\title{
Validez y fiabilidad del Inventario de Ideación Suicida Positiva y Negativa - PANSI, en estudiantes colombianos"
}

\author{
Validity and Reliability of the Positive and Negative Suicidal \\ Ideation Inventory, in Colombian Students
}

Recibido: junio 16 de 2009 | Revisado: agosto 20 de 2009 | Aceptado: agosto 30 de 2009

\author{
Fredy HeRnÁN Villalobos-Galvis ** \\ Departamento de Psicología, Grupo Psicología y Salud,
}

Universidad de Nariño

Para citar este artículo. Villalobos-Galvis, F.H. (2010). Validez y fiabilidad del Inventario de Ideación Suicida Positiva y Negativa-PANSI, en estudiantes colombianos. Universitas Psychologica, 9 (2), 509-520.

* Este trabajo fue financiado por el Sistema de Investigaciones de la Universidad de Nariño (Colombia). El autor declara que no tiene ningún conflicto de intereses y que el estudio fue aprobado por el comité de ética de la Universidad de Nariño, garantizando el cumplimiento de disposiciones bioéticas y legales establecidas para este tipo de investigaciones.

** Correo electrónico: fhvillalobos@udenar.edu.co

RESUMEN

Para conocer las características psicométricas de una versión en español del Inventario de Ideación Suicida Positiva y Negativa-PANSI, se realizó un estudio de tipo instrumental, con la participación de 643 estudiantes de colegios y universidades de San Juan de Pasto (Colombia). El análisis factorial exploratorio mostró una estructura bifactorial que explicaba el 64\% de la varianza. El primer factor correspondió a ideas suicidas negativas, mientras que el segundo, abarcó pensamientos protectores ante el suicidio. La escala total y las subescalas tuvieron altos valores de consistencia interna. El PANSI tuvo relaciones significativas con medidas de depresión, desesperanza, ideas suicidas y autoestima, y con indicadores de gravedad de la conducta suicida. Se concluye que el PANSI tiene buenas propiedades psicométricas, razón por la cual podría ser utilizada con estudiantes adolescentes y jóvenes. Palabras clave autor

Ideas suicidas, suicidio, escala de medición, análisis factorial, validez, fiabilidad, estudiantes, Colombia.

Palabras clave descriptores

Estudiantes, intento de suicidio, psicología, suicidio, San Juan de Pasto, Colombia.

\begin{abstract}
A B S T R A C T
With the aim of knowing the psychometric characteristics of a Spanish version of the Positive and Negative Suicidal Ideation Inventory-PANSI, an instrumental study was developed. Its application required the participation of 643 students of high schools and universities of San Juan de Pasto (Colombia). The exploratory factor analysis showed a bi-factor structure, that accounted for $64 \%$ of variance. The first factor corresponded to negative suicidal thoughts, while the second one included protective thoughts against suicide. The total scale and the sub-scales had high values of internal consistency. Significant relationships were found between PANSI and measures of depression, hopelessness, suicidal thoughts and self-esteem, and with severity indicators of suicidal behavior. It was concluded that PANSI has adequate psychometric properties, making it suitable to be used with adolescents and young students.

Key words author

Suicidal Ideation, Suicide, Measurement Scale, Factor Analysis, Validity, Reliability, Students, Colombia.

Key words plus

Students, Suicide, Attempted, Psychology, Suicide, San Juan de Pasto (Colombia).
\end{abstract}


El suicidio es un fenómeno humano particularmente complejo, no solo por sus causas, sino además por las dificultades que implican su definición y su atención. En el estudio del suicidio (Suicidology) se propone el concepto de espectro suicida, en el cual se plantea que la conducta suicida pasa por estadios de menor a mayor gravedad, iniciando por las ideas, pasando por las tentativas y culminando con la muerte por suicidio. Se habla de ideas suicidas cuando un sujeto persistentemente piensa, planea o desea cometer suicidio, haciendo algún plan e identificando los medios necesarios para conseguirlo (Mingote, Jiménez, Osorio \& Palomo, 2004). Por su parte, un intento de suicidio es definido como cualquier acto deliberado, con resultado no fatal, que pretende causar o realmente causa daño a sí mismo o que sin la intervención de otros habría sido así o que consiste en ingerir una sustancia en exceso de la dosis generalmente reconocida o de la prescrita terapéuticamente, y el cual es realizado con el fin de realizar cambios, a través de las consecuencias objetivas o subjetivas que tal acto pueda llegar a tener (De Leo et al., 2004). Por último, O'Carroll et al. (1996) definen al suicidio como "la muerte debida a lesiones, envenenamiento o sofocación, donde hay evidencia (explícita o implícita) de que la lesión fue auto-inflingida y que la intención del occiso era matarse" (pp. 246-247).

Según la OMS (2003), la conducta suicida en sus distintas formas representa un problema de salud pública internacional, ya que se encuentra dentro de las diez principales causas de mortalidad general, y entre las tres primeras causas de muerte en el grupo de adolescentes y adultos jóvenes.

La presencia de conductas suicidas en estudiantes de educación secundaria y universitaria de Latinoamérica, ha sido reportada por diferentes investigaciones, en las cuales se han hallado indicadores de ideación suicida que oscilan entre el 8 y el 25\% de los casos, mientras que los intentos de suicidio varían entre el 8 y el 12\% (GonzálezForteza et al., 1998a, 1998b, 2002; Haseitel et al., 2004; Rodríguez et al., 2006).

En Colombia, las tasas de suicidio durante los años 1997 a 2007 oscilaron entre 4 y 5 muertes por cada 100.000 habitantes, siendo el período entre los 15 y 44 años el que presenta los mayores porcentajes de casos (González, 2007), demostrándose que es un problema que tiene sus inicios en la adolescencia y la edad adulta joven.

En el plano del municipio de Pasto, de acuerdo con los informes anuales emitidos por el Observatorio del Delito ${ }^{1}$, durante el quinquenio 2003. 2007, se registraron 229 muertes por suicidio. Entre ellas, se destaca la mayor frecuencia de casos de hombres, en una proporción 2:1 frente a las mujeres. De igual forma, la tasa por 100.000 habitantes ha fluctuado entre 8.6 (en 2005) y 14.2 (en 2006) (véase Tabla 1), las cuales resultan significativamente altas en comparación con la tasa nacional. Ahora bien, el 64.6\% las muertes por suicidio, así como el 70\% de los intentos, se dan en personas con edades entre 15 y 29 años, lo que corresponde a la etapa evolutiva de la adolescencia media y tardía, y a la edad adulta joven, en edades que típicamente se asocian a la finalización de la formación educativa secundaria o universitaria y al inicio de la actividad económica laboral.

\section{TABLA 1}

Casos de muerte por suicidio en el municipio de Pasto (2003-2007)

\begin{tabular}{lcccccc}
\hline & \multicolumn{6}{c}{ Año } \\
\cline { 2 - 7 } \multicolumn{1}{c}{ Sexo } & 2003 & 2004 & 2005 & 2006 & 2007 & Total \\
\hline Hombre & 28 & 26 & 23 & 41 & 39 & 157 \\
$\%$ & 57.14 & 63.41 & 69.70 & 74.55 & 76.47 & 68.56 \\
\hline Mujer & 21 & 15 & 10 & 14 & 12 & 72 \\
$\%$ & 42.86 & 36.59 & 30.30 & 25.45 & 23.53 & 31.44 \\
\hline Total & 49 & 41 & 33 & 55 & 51 & 229 \\
Tasa & 13.1 & 10.9 & 8.6 & 14.2 & 12.9 & \\
\hline
\end{tabular}

Fuente: elaboración propia.

1 El observatorio de muertes por causa externa, denominado "Observatorio del Delito del municipio de Pasto" es una estrategia de origen gubernamental, con participación multisectorial, cuyo objetivo es "consolidar en el municipio (...) un sistema de vigilancia de eventos violentos a partir de la implementación de un sistema de información georreferenciado que de manera permanente, oriente y apoye el desarrollo de políticas de prevención y control como también que permitan la verificación del comportamiento del fenómeno con miras a la elaboración de una política local de prevención de violencia articulada al Plan de Desarrollo Municipal" (CISALVA, 2008, p. 27). 
En un estudio previo realizado en esta misma ciudad (Villalobos-Galvis, 2009) se encontró que alrededor de un 30\% de los estudiantes de secundaria y universidad, ha presentado alguna conducta suicida no letal durante el transcurso de su vida, del cual un $8 \%$ fueron intentos de suicidio y un $22 \%$ ideas o planes suicidas. También se halló que el $80 \%$ de los estudiantes que tuvieron un intento, no reportaron este hecho a ninguna persona, ni acudieron por ayuda a ninguna institución, por lo cual se infiere que es muy factible que las cifras reales de casos de intentos de suicidio sean aún mayores que las oficialmente reportadas en el municipio de Pasto.

A partir de la información epidemiológica, diferentes instancias gubernamentales y académicas del municipio consideran que la conducta suicida es un importante problema de salud pública, que requiere del aporte de diferentes agentes sociales para su prevención.

Una de las principales estrategias de prevención es la identificación temprana de casos, a través de la evaluación de la presencia e intensidad de las ideas suicidas, las cuales han demostrado ser la forma más común de conducta suicida y, a menudo, el precursor más importante de las subsiguientes conductas autolesivas de mayor gravedad (Konick \& Gutiérrez, 2005).

Diversas investigaciones han demostrado ampliamente que la ideación suicida tiene correlaciones positivas con variables de riesgo, tales como depresión, desesperanza, eventos vitales estresantes, etc.; así como relaciones negativas con variables protectoras para la salud mental, tales como apoyo social, autoestima, razones para vivir y solución de problemas sociales (Bhar et al., 2008; Clum et al., 1997; Chioqueta \& Stiles, 2007; Field et al., 2001; González-Forteza et al., 2003; Gutiérrez et al., 2002; Konick \& Gutiérrez, 2005; Lieberman et al., 2005; Nugent \& Williams, 2001; VillalobosGalvis, 2009; Wild et al., 2004).

El Inventario de Ideación Suicida Positiva y Negativa (Positive and Negative Suicide Ideation PANSI), fue desarrollado por Osman, Gutiérrez, Kopper, Barrios y Chiros (1998) con el propósito de medir, de manera simultánea, factores de riesgo y de protección frente a las ideas suicidas, siendo uno de los instrumentos que responde a la pretensión de identificar tempranamente casos de conductas suicidas. No obstante su posible utilidad, no se conocen estudios en los cuales el PANSI haya sido traducido y adaptado para la población colombiana, razón por la cual el interés de este trabajo fue determinar la validez y la fiabilidad de una versión en español, en estudiantes de algunos colegios y universidades de San Juan de Pasto.

\section{Método}

\section{Tipo de estudio}

El presente trabajo es un estudio de tipo instrumental, pues está encaminado a la adaptación del PANSI y al estudio de sus propiedades psicométricas (Montero \& León, 2007). Para ello se hizo uso del programa SPSS 13 para Windows.

\section{Instrumentos}

Inventario de Ideación Suicida Positiva y Negativa (Positive and Negative Suicidal Ideation - PANSI)

El instrumento fue adaptado, para este estudio, por medio de la técnica de traducción simple. El PANSI (Osman et al., 1998) es un cuestionario de 14 ítems, 6 de ideación suicida positiva (factores protectores) y 8 de ideación suicida negativa (factores de riesgo), que son evaluados en el marco de las últimas dos semanas y en los que se pregunta qué tan a menudo la persona ha presentado cada uno de los 14 pensamientos. La persona debe responder haciendo uso de una escala de 5 puntos que oscilan entre 0 (nunca) y 4 (siempre). Este inventario ha mostrado coeficientes de consistencia interna superiores a 0.8 y buena evidencia de validez de constructo (concurrente y discriminante), tanto en muestras clínicas como no-clínicas (Osman et al., 2003). Este inventario consta de dos escalas factorialmente derivadas: Ideación positiva e Ideación negativa. El trabajo original sobre el PANSI se llevó a cabo en dos estudios. En el primero, los autores encontraron una estructura 
bifactorial, en una muestra normativa de 450 estudiantes universitarios entre los 18 y 25 años de edad. El segundo estudio implicó a 286 estudiantes de pregrado con edades entre los 18 y 48 años, en el cual el análisis factorial confirmatorio validó la estructura de dos factores propuesta en el estudio 1 (Osman et al., 1998).

Los análisis factoriales confirmatorios que se han hecho sobre la prueba, demostraron la validez factorial de la escala, así como su estructura bifactorial, en diferentes grupos raciales (Muehlenkamp et al., 2005); estudiantes de secundaria (Osman et al., 2003) y pacientes internos adolescentes (Osman et al., 2002). También se ha encontrado evidencia de validez concurrente y predictiva en diferentes muestras de estudio (Muehlenkamp et al., 2005).

De otro lado, con el fin de contar con evidencias para la validez de constructo, se hizo uso de las siguientes escalas de medida adicionales.

Escala de Depresión del Centro de Estudios Epidemiológicos (Center of Epidemiological Studies - Depression Scale - CESD)

Es una escala compuesta por 20 ítems, con 4 opciones de respuesta, que evalúa los síntomas de depresión durante las últimas 2 semanas, en población general. Los síntomas se agrupan en cuatro escalas derivadas factorialmente: ánimo depresivo, síntomas somáticos, problemas sociales y bienestar. El CES-D ha demostrado alta consistencia interna, fiabilidad test-retest, validez concurrente y validez de constructo (Radloff, 1977). En estudios de Villalobos-Galvis $(2006,2010)$ se demostró que esta escala tiene adecuada validez factorial y consistencia interna alta, al ser usada en estudiantes de secundaria y universidad de la ciudad.

Escala de Desesperanza de Beck (Beck Hopelessness Scale-BHS)

La escala (Beck, Weissman, Lester \& Trexler, 1974) consta de 22 ítems, con formato de respuesta de falso y verdadero, los cuales evalúan los tres aspectos más importantes de la desesperanza: sentimientos acerca del futuro, pérdida de la motivación y expectativas negativas. Posee muy buenos datos de fiabilidad y obtiene resultados positivos respecto a su validez (González-Macip et al., 2000). Esta prueba fue adaptada y validada en estudiantes de San Juan de Pasto, demostrando una adecuada validez factorial y una alta consistencia interna (alfa de Cronbach $=0.83)($ Villalobos-Galvis, 2006).

Escala de autoestima de Rosenberg (AER)

Es uno de los instrumentos más utilizados en la medición global de la autoestima. Fue desarrollada por Rosenberg (1965), originalmente para la evaluación de la autoestima en adolescentes e incluye 10 ítems cuyos contenidos se centran en los sentimientos de respeto y aceptación de sí mismo. La mitad de los ítems están redactados de manera positiva y la otra mitad de manera negativa. Es una escala que ha demostrado una buena fiabilidad y validez en diferentes contextos, incluyendo el de la población de estudio (Vázquez, Jiménez \& Vázquez, 2004; Villalobos-Galvis, 2006).

Escala de ideación suicida (IS-CESD)

Es una escala compuesta por tres reactivos concernientes a sentimientos y pensamientos sobre la propia muerte, con el mismo formato de respuesta del CES-D, en donde a mayor puntuación, mayor ideación suicida. Desarrollada por Roberts (1980), se contó con la adaptación mexicana llevada a cabo por Medina-Mora et al. (1994). En un estudio llevado a cabo por González-Forteza (1992), los tres reactivos mostraron una alta consistencia interna (alfa de Cronbach de 0.78), así como congruencia conceptual al agruparse en un solo factor con valor propio mayor a 1, en un análisis factorial exploratorio, explicando el $70.1 \%$ de la varianza total.

\section{Participantes}

Para este estudio se contó con el apoyo voluntario de instituciones de educación secundaria y superior de la ciudad de San Juan de Pasto. Se llevó a cabo un muestreo no probabilístico de sujetos vo- 
luntarios, por cuotas de sexo y años escolares. En virtud de lo anterior, participaron 643 estudiantes de 6 colegios y 2 universidades de la ciudad de San Juan de Pasto. En este grupo se contó con una participación similar de hombres y de mujeres (320 y 323, respectivamente). La edad de los participantes fluctuó entre los 10 y los 30 años, con una media de 16.8 años y una desviación estándar de 4.34 . Las edades más frecuentes se presentaron entre los 13 y 15 (más de 10.4\%) y las menos frecuentes fueron los mayores de 27 años (menos del 1\%). El grupo estudiado abarcó a estudiantes de $6^{\mathrm{O}}$ grado de bachillerato a 50 año de universidad. El 55.5\% estaban matriculados en grados de bachillerato y el otro $44.5 \%$ eran estudiantes universitarios. Se consiguió una participación prácticamente similar entre estudiantes de instituciones del sector oficial (52.6\%) y del sector privado (47.4\%).

\section{Resultados}

\section{Análisis factorial exploratorio (AFE)}

Con el fin de evaluar la validez de la versión en español del PANSI, se decidió utilizar el procedimiento del análisis factorial exploratorio (AFE), pues se quería conocer si los ítems tendían a agruparse en los mismos factores propuestos en el estudio original con la versión inglesa (Osman et al., 1998).

De esta manera, se procedió a comprobar los supuestos para la adecuada aplicación del AFE, por lo cual se realizó la prueba de Kaiser-Meyer-Olkin, de la que se obtuvo un coeficiente de 0.932 , mientras que la prueba de esfericidad de Bartlett resultó significativa $\left(\chi^{2}=5581.48 ; g l=91 ; p<.001\right)$ y las comunalidades oscilaron entre .29 (ítem 2) y.76 (ítems 5 y 9); elementos que ofrecen certezas frente a la viabilidad del uso de la técnica en este caso.

Al realizar el AFE con la técnica de componentes principales, se replica la estructura bifactorial propuesta por Osman et al. (1998, 2002, 2003), en la que estos dos componentes explican el 63.8\% de la varianza. De igual manera, y siguiendo los planteamientos del grupo de Osman et al. (1998,
2002, 2003) y Muehlekamp et al., (2005), se llevó a cabo una rotación oblicua por la técnica Oblimin con Normalización de Kaiser, la cual produjo las estructuras presentadas en las Tablas 1 y 2, luego de 3 iteraciones.

En la Tabla 2 aparecen los ítems que conforman el primer componente, el cual reproduce el factor de ideas suicidas negativas formulado por Osman et al. (1998), e implica a las ideas suicidas propiamente dichas. Como se puede observar, los pensamientos que se abarcan hacen referencia no solamente a la consideración del acto suicida, sino que formulan las posibles razones que lo justificarían, tales como el fracaso, la desesperanza, la baja autoefícacia, la frustración o la tristeza.

\section{TABLA 2}

Ítems del factor de ideas suicidas negativas

\begin{tabular}{|c|c|}
\hline Ítem & $\begin{array}{l}\text { Carga } \\
\text { Factorial }\end{array}$ \\
\hline $\begin{array}{l}\text { 5. ¿Pensaste en matarte porque no pudis- } \\
\text { te hacer algo que era muy importante } \\
\text { en tu vida? }\end{array}$ & .880 \\
\hline $\begin{array}{l}\text { 10. ¿Pensaste que tus problemas eran tan } \\
\text { graves que la única opción que tenías } \\
\text { era suicidarte? }\end{array}$ & .872 \\
\hline $\begin{array}{l}\text { 7. ¿Pensaste en matarte porque no en- } \\
\text { contraste una solución a un problema } \\
\text { personal? }\end{array}$ & .861 \\
\hline $\begin{array}{l}\text { 9. ¿Pensaste en matarte porque viste que } \\
\text { tu vida era un fracaso? }\end{array}$ & .859 \\
\hline $\begin{array}{l}\text { 11. ¿Te sentiste tan solo(a) o tan triste que } \\
\text { querías matarte para así terminar con } \\
\text { ese sufrimiento? }\end{array}$ & .836 \\
\hline $\begin{array}{l}\text { 3. ¿Pensaste en matarte porque no tenías } \\
\text { esperanza en el futuro? }\end{array}$ & .834 \\
\hline $\begin{array}{l}\text { 1. ¿Has considerado seriamente matarte } \\
\text { porque no pudiste cumplir con lo que } \\
\text { otras personas esperaban de ti? }\end{array}$ & .821 \\
\hline $\begin{array}{l}\text { 4. ¿Te sentiste tan triste por tu relación } \\
\text { con alguien importante, que quisiste } \\
\text { estar muerto? }\end{array}$ & .664 \\
\hline
\end{tabular}

Fuente: elaboración propia.

Por su parte, en la Tabla 3 se puede observar que el segundo factor corresponde con los ítems 
de ideación suicida positiva (Osman et al., 1998) o pensamientos de protección frente al suicidio. En ellos se destaca la presencia de razones protectoras tales como el sentido de control, la autoconfianza, las expectativas positivas, la alegría y la satisfacción con la vida.

TABLA 3

Ítems del factor de ideas suicidas positivas

\begin{tabular}{lc}
\hline \multicolumn{1}{c}{ Ítem } & $\begin{array}{c}\text { Carga } \\
\text { Factorial }\end{array}$ \\
\hline $\begin{array}{l}\text { 12. iTuviste confianza en las capacidades } \\
\text { que tenías para enfrentar la mayoría de } \\
\text { problemas de tu vida? }\end{array}$ & .848 \\
14. iTuviste confianza en lograr tus metas & .808 \\
en el futuro? & \\
6. $\begin{array}{l}\text { iTuviste esperanza en el futuro porque } \\
\text { las cosas estaban saliendo como tu } \\
\text { querías? }\end{array}$ & .777 \\
8. iTe sentiste alegre porque te estaba & \\
yendo bien en el colegio o en el & .771 \\
trabajo? & \\
13. iSentiste que valía la pena vivir la vida? & .666 \\
2. iHas sentido que tenías el control de la & \\
mayoría de las situaciones de tu vida? & .546 \\
\hline
\end{tabular}

Fuente: elaboración propia.

El uso de la técnica de rotación oblicua, implica que los dos factores identificados no son completamente independientes, por lo cual resultaba pertinente conocer si existe alguna relación entre ellos. Por esta razón, se calculó el coeficiente de correlación que fue igual a 0.44. Considerando que los ítems del factor de ideas positivas se califican de manera inversa, el signo de la correlación implica que la alta presencia de ideas de suicidio podría estar relacionada con una baja presencia de ideas positivas, y viceversa. De otro lado, el coeficiente de correlación, aunque tiene un valor moderado, es estadísticamente significativo $(p<.001)$, lo que implica que si bien estarían evaluando dos dimensiones de un mismo constructo, tales dimensiones no serían la misma, sino que guardarían algunas diferencias importantes.

\section{Validación del modelo factorial según variables sociodemográficas}

Tal como lo plantea Bisquerra (1989), una forma de validar los resultados de un análisis factorial exploratorio es comparar las soluciones factoriales entre subgrupos de la población. Por ello, se realizaron diferentes análisis factoriales exploratorios, haciendo uso de la misma técnica, en los subgrupos de mujeres, hombres, bachilleres y universitarios. En todos ellos se encontraron dos factores con valores propios mayores a 1 , comprobando la estructura bifactorial propuesta para la prueba (véase Tabla 3). De igual forma, se halló que la varianza explicada osciló entre el 60 y el 68\%, a través de los diferentes subgrupos, con lo que se corrobora la importancia explicativa de los dos factores hallados.

\section{TABLA 4}

Estadísticos generales del análisis factorial por subgrupos de la muestra

\begin{tabular}{lcccc}
\hline \multicolumn{1}{c}{ Estadístico } & $\begin{array}{c}\text { Muje- } \\
\text { res }\end{array}$ & $\begin{array}{c}\text { Hom- } \\
\text { bres }\end{array}$ & $\begin{array}{c}\text { Bachi- } \\
\text { lleres }\end{array}$ & $\begin{array}{c}\text { Univer- } \\
\text { sitarios }\end{array}$ \\
\hline $\begin{array}{l}\text { Comunalidad } \\
\text { mínima }\end{array}$ & .313 & .290 & .287 & .338 \\
$\begin{array}{l}\text { Comunalidad } \\
\text { máxima }\end{array}$ & .793 & .791 & .751 & .857 \\
$\begin{array}{l}\text { No. de com- } \\
\text { ponentes con } \\
\text { valores propios }\end{array}$ & 2 & 2 & 2 & 2 \\
$>1$ & & & & \\
$\begin{array}{l}\text { Varianza expli- } \\
\text { cada }\end{array}$ & $66.58 \%$ & $59.94 \%$ & $61.74 \%$ & $67.98 \%$ \\
$\begin{array}{l}\text { Correlación } \\
\text { entre factores }\end{array}$ & .474 & .378 & .422 & .433 \\
\hline
\end{tabular}

Fuente: elaboración propia.

Finalmente, se encontraron valores de correlación moderados entre los componentes de la escala, manteniendo la propuesta de que si bien dicha relación es estadísticamente significativa $(p<.01)$ (indicando que hay asociación entre tales factores), los moderados valores absolutos de la correlación (.38 a .47) también indican que no están evaluando exactamente los mismos elemen- 
tos (véase Tabla 3), confirmando con ello la idea de que son dos factores diferentes que miden un mismo atributo: la ideación suicida.

Igualmente, se llevó a cabo un análisis de la invarianza factorial de las diferentes soluciones obtenidas, a través del cual se observó que el primer componente emergente siempre fue el de las ideas suicidas negativas y que en todos los subgrupos poblacionales, los ítems siempre cargaron en el mismo factor que en el estudio de validación original realizado por Osman et al. (1998). Estos datos permiten comprobar que la estructura factorial de la prueba, en su versión en español, se mantiene en comparación con la hallada en el estudio original.

\section{Consistencia interma de la prueba total y de las subescalas}

Las correlaciones moderadas (en intensidad) pero estadísticamente significativas, obtenidas entre los factores que componen la escala, permiten hacer uso del concepto de consistencia interna, tanto para la escala total, como para cada uno de los factores identificados, como una adecuada medida de fiabilidad. Al realizar el cálculo del respectivo coeficiente para la prueba total, se encontró que el estadístico alpha de Cronbach fue .899, indicando un buen nivel de consistencia interna. Las correlaciones ítem-escala fueron significativas $(p<.01)$ en todos los casos y fluctuaron entre .37 (ítem 2) y .72 (ítem 9), demostrando una importante interrelación de los ítems que componen la prueba.

Analizando la consistencia interna del primer factor (ideas negativas), se obtuvo un coeficiente alfa de Cronbach de .931. Se pudo determinar que los ítems tienen una alta correlación con la subescala $(p<.01)$, expresada en coeficientes que varían entre .59 y .82 , lo cual demuestra que existe una alta covariación entre ellos. Finalmente, el segundo factor (ideas positivas), obtuvo un coeficiente alfa de Cronbach de .836. En este caso también se observó que los coeficientes de correlación ítemprueba varían entre .411 y .717 $(p<.01)$, demostrando una buena interrelación de cada uno de ellos con la suma de las puntuaciones de los demás ítems. En conclusión, se encontró que tanto la escala total como cada una de las subescalas del PANSI poseen una buena consistencia interna.

\section{Evidencias de validez de constructo.}

Para comprobar la validez de constructo de la prueba, se esperaba, de acuerdo con la teoría, que el PANSI tuviera relaciones positivas con las medidas de variables de riesgo y negativas con las de protección.

En la Tabla 4 se presentan los coeficientes de correlación por rangos de Spearman, entre medidas de depresión (CESD), desesperanza (BHS), autoestima (AER) e ideación suicida (IS CESD), con las subescalas y la escala total del PANSI. De acuerdo con los p-valores, todas las correlaciones son altamente significativas $(p<.001)$. En ese sentido, la escala y las subescalas del PANSI tienen relaciones positivas con medidas de depresión, desesperanza e ideas suicidas, mientras que tienen relaciones negativas con la escala de autoestima. Un elemento interesante es que la escala total del PANSI muestra las mayores correlaciones con las demás variables, mientras que el mayor valor absoluto de los coeficientes de correlación, corresponde a la asociación entre la escala de ideas suicidas de Roberts (IS-CESD) y la subescala de ideas negativas (o ideas suicidas propiamente dichas).

\section{TABLA 5}

Correlaciones entre escalas del PANSI y variables relevantes

\begin{tabular}{lcccc}
\hline & CESD & BHS & AER & IS-CESD \\
\hline $\begin{array}{l}\text { PANSI Ideas } \\
\text { Negativas }\end{array}$ & .476 & .377 & -.371 & .571 \\
PANSI Ideas & .471 & .462 & -.475 & .324 \\
Positivas & .534 & .478 & -.495 & .472 \\
PANSI Total &
\end{tabular}

Nota: CESD = Escala de Depresión del Centro de Estudios Epidemiológicos; $\mathrm{BHS}=$ Escala de Desesperanza de Beck; AER = Escala de Autoestima de Rosenberg; IS-CESD = Escala de Ideas Suicidas de Roberts.

Fuente: elaboración propia. 
Por último, como un elemento adicional de evidencia de validez de constructo de la escala, se esperaba que el PANSI presentara un comportamiento ajustado al concepto de espectro suicida, es decir, que a medida que aparecieran conductas suicidas más graves, las puntuaciones de la escala fueran aumentando.

Para probar esta hipótesis se llevó a cabo un análisis de varianza no paramétrico, haciendo uso de la prueba Kruskal Wallis (Siegel \& Castellán, 2007). En este análisis, se consideraba a la conducta suicida como variable de clasificación, la cual se evaluaba a través de la pregunta: "iAlguna vez tú mismo te has herido, cortado, intoxicado o hecho daño a propósito, con el fin de quitarte la vida?" ante la cual la personas podían marcar "no, nunca lo he hecho" (ninguna), "sólo lo pensé" (ideas), "estuve a punto de hacerlo" (planes) o "sí lo hice" (intento). Esta pregunta ha sido validada en estudios epidemiológicos realizados por Gonzalez-Forteza et al. (1998a, 1998b, 2000, 2003), demostrando que es un buen descriptor de la prevalencia durante la vida de conductas suicidas en adolescentes y adultos jóvenes.

El estadístico de prueba calculado permitió rechazar la hipótesis nula y asumir que sí existen diferencias significativas de las puntuaciones totales en el PANSI, entre los diferentes grupos de conductas suicidas $\left(\chi^{2}=135.87, g l=3, p<001\right)$. Así pues, en la Tabla 5 se observa que las personas que no reportaron ninguna conducta suicida tienen los menores valores de la escala total y que a medida que el espectro suicida se va agravando (ideas, planes e intentos), los valores de la escala total van aumentando consecuentemente. De esta forma, quienes han tenido intentos de suicidio durante su vida, son los que tienen mayores puntuaciones de ideación suicida en la escala total.

\section{Discusión y Conclusiones}

El propósito del presente trabajo era determinar la validez y la fiabilidad de una versión en español del Inventario de Ideación Suicida Positiva y Negativa - PANSI, en estudiantes de colegios y universidades de San Juan de Pasto. Dentro de los principales resultados obtenidos en esta investigación, se encuentran: a) que el PANSI mantiene la estructura bifactorial propuesta para la versión inglesa, b) que presenta una buena fiabilidad, de acuerdo con los coeficientes de alpha de Cronbach calculados tanto para la escala total (.899), como para las subescalas (.931 para ideas negativas y .836 para ideas positivas); y, c) que las puntuaciones de la escala están asociadas con medidas de desesperanza, depresión, ideas suicidas y autoestima, así como con el concepto de espectro suicida, demostrando de esta manera su validez de constructo.

Uno de los intereses que surgen cuando se hacen adaptaciones culturales de instrumentos de medida, es evaluar el grado de estabilidad en la estructura de la prueba, a través de las diferentes

TABLA 6

Puntuaciones en la escala total de acuerdo con tipo de conducta suicida

\begin{tabular}{lccccc}
\hline $\begin{array}{c}\text { Tipo de conducta } \\
\text { suicida }\end{array}$ & $\mathbf{n}$ & $\%$ & $\begin{array}{c}\text { Rango medio de la } \\
\text { escala total }\end{array}$ & Media & DT \\
\hline Ninguna & 420 & 65.6 & 293.34 & 9.12 & 9.23 \\
Ideas & 127 & 19.8 & 349.75 & 11.09 & 9.03 \\
Planes & 28 & 4.4 & 367.77 & 12.64 & 10.81 \\
Intentos & 65 & 10.2 & 434.11 & 15.63 & 10.328 \\
Total & 640 & 100 & & 10.25 & 9.544 \\
\hline
\end{tabular}

Nota: Se excluyeron 3 casos por omisión de respuestas.

Fuente: elaboración propia. 
versiones que se hagan de la misma. En virtud de ello, este trabajo pretendía observar la estructura factorial de la escala, con miras a validar el carácter bidimensional con el que fue creada. Para ello se hizo uso de la técnica del análisis factorial exploratorio, la cual demostró, una vez más, la presencia de dos factores claros en la prueba, los cuales explicaban el $64 \%$ de su variabilidad.

Una forma de validar los resultados de un análisis factorial exploratorio es la confirmación de la invarianza factorial, a través de diferentes subgrupos de la muestra estudiada (Bisquerra, 1989). Así pues, en el presente estudio se hicieron comparaciones entre hombres y mujeres, y entre estudiantes de bachillerato y de universidad. En todas las muestras se repitió la misma solución bifactorial, lo que valida los hallazgos de la muestra total.

En cuanto a la solución factorial, el primer componente saliente (tanto para la muestra total, como para los subgrupos) fue el de las ideas suicidas negativas, o ideas suicidas propiamente dichas, en el que saturaban de manera significativa 8 ítems, en cuyo contenido se esbozaba que la persona consideraba al suicidio como una posibilidad ante una serie de circunstancias negativas que le afectaban de manera significativa. Este factor también surgió como primer componente en los diferentes estudios que ha realizado el grupo de Osman et al. (1998, 2002, 2003) y Muehlekamp et al., (2005), validando que es la estructura más sobresaliente de la prueba.

El segundo factor, conocido como ideas suicidas positivas o ideas protectoras frente al suicidio, estaba compuesto por 6 ítems que se caracterizaban por presentar pensamientos de autoeficacia y esperanza hacia el futuro, y por los cuales se desechaba la posibilidad del suicidio. Tales ítems fueron los mismos que cargaron en el segundo factor en los estudios de validación (Muehlekamp et al., 2005; Osman et al. 1998, 2002, 2003), por lo que también se comprueba la pertinencia de esta escala en la versión en español del PANSI.

Los resultados del análisis factorial, con el uso de la técnica de rotación oblicua, así como el cálculo del coeficiente de correlación entre las dos subescalas de la prueba, permiten suponer que son medidas independientes de un mismo constructo, pero que sus mediciones resultan complementarias a la hora de evaluar a la ideación suicida. Estos resultados concuerdan con los encontrados en otras investigaciones sobre esta misma prueba (Muehlekamp et al., 2005; Osman et al., 1998, 2002, 2003).

Por otro lado, los altos valores de los coeficientes de fiabilidad, tanto de la escala total, como de las dos subescalas, apoyan la idea que los ítems evalúan un mismo atributo, con dos dimensiones componentes. También, demuestran que los ítems están interrelacionados, por lo que las medidas obtenidas en cualquiera de ellos, se relacionan significativamente con las obtenidas en los demás. Esto apoya el uso de la escala, al asumirse que es un instrumento de medición consistente y con alta coherencia interna.

Por su parte, los hallazgos de validez de constructo tienen un alto valor para este estudio, ya que el PANSI, tanto en su escala total como en sus subescalas, demostró relaciones muy significativas con variables que la literatura ha comprobado que están significativamente asociadas, a saber: la depresión (Clum et al., 1997; Field et al., 2001; Konick \& Gutiérrez, 2005; Pollock \& Williams, 2004; Wild et al., 2004), la desesperanza (Clum et al., 1997; Chioqueta \& Stiles, 2007; Schotte \& Clum 1987) y la autoestima (Bhar et al., 2008; Chioqueta \& Stiles, 2007; Lieberman et al., 2005; Wild et al., 2004). De esta manera, se comprueba que las medidas que ofrece el PANSI guardan coherencia teórica con lo hallado en la literatura científica sobre las ideas suicidas.

Un comentario especial merece la relación entre el PANSI y la escala de ideación suicida ISCESD (Roberts, 1980), pues se ve cómo la subescala de ideas negativas es la que tiene una mayor relación lineal con esta última. En ese sentido hay que reconocer que tanto la subescala de ideas negativas como la IS-CESD, son instrumentos que evalúan, de manera manifiesta, la presencia de ideas suicidas, razón por la cual sus coeficientes de correlación son altos; mientras que la escala total del PANSI toma en consideración a la otra dimensión, a saber, los pensamientos protectores ante el 
suicidio, lo cual hace que ya no se esté hablando propiamente del mismo fenómeno.

La importante relación entre la escala de autoestima y la subescala de ideas suicidas positivas, demostraría que efectivamente ésta evalúa un factor de protección, pues como plantean Cabrera y Castillo (2008), los factores de protección ante las ideas suicidas presentan altos coeficientes de correlación múltiple, es decir, que el aumento en los valores de uno de ellos está asociado con una elevación generalizada de los demás. Así pues, se asume que la alta presencia de autoestima se relaciona con las ideas suicidas positivas, tanto por el factor protector que en sí representa la autoestima, como por las relaciones que ésta última tiene con otras variables tales como el apoyo social, las razones para vivir o la solución de problemas (Cabrera \& Castillo, 2008; Clum et al., 1997; Groholt et al., 2005; Lieberman et al., 2005; Pollock \& Williams, 2004).

De otro lado, se observó que el 35\% de los estudiantes reportó alguna conducta suicida durante sus vidas y, específicamente, un 20\% tuvo pensamientos de suicidio. Estos datos se mantienen dentro de la tendencia encontrada en otro estudio en el mismo municipio (Villalobos-Galvis, 2009), así como lo hallado en otros países (González-Forteza et al. 2003; González-Macip et al., 2001), en los cuales se plantea la hipótesis de que las ideas suicidas pueden ser productos cognitivos "normales", en el sentido de que un buen porcentaje de personas las tiene a lo largo de su vida. Sin embargo, es la presencia excesiva, intensa, permanente y generalizada la que determina su potencial amenazante, para el paso a una conducta suicida más grave, tal como el intento o el suicidio consumado.

Por último, es importante reconocer cómo las puntuaciones de la escala total del PANSI muestran un comportamiento diferencial entre los diversos grupos de la conducta suicida. En ese sentido se demostraría la validez de la escala en relación con el concepto de Espectro Suicida, de amplio uso en el estudio de las conductas suicidas (Villalobos-Galvis, 2007), y del papel que juegan los pensamientos de suicidio en él, pues se confirma cómo a medida que aumenta la gravedad de la conducta suicida, se da una mayor presencia de ideas de muerte autoinflingida (Konick \& Gutiérrez, 2005).

En este sentido, el hecho de que las medidas del PANSI covaríen con la intensidad de la conducta suicida, es un hallazgo de gran relevancia práctica, pues demostraría que una alta puntuación en la escala total puede ser un importante indicador de alto riesgo suicida. Así pues, se sugiere la realización de estudios epidemiológicos en los cuales se valide su uso en acciones de detección temprana de casos de estudiantes con diferentes niveles de riesgo. Una ventaja adicional que tendría el uso de esta escala es que por su estructura, no sólo evaluaría el riesgo, sino que además puede dar indicaciones de los niveles de protección con los que cuenta la persona, frente al hecho de pensar en el suicidio como una opción posible.

En conclusión, en este estudio se demuestra que la versión en español del Inventario de Ideas Suicidas Positivas y Negativas (PANSI) es un instrumento con excelentes propiedades psicométricas que permiten sugerir su uso en estudiantes de colegios y universidades.

\section{Referencias}

Beck, A., Weissman, A., Lester, D. \& Trexler, L. (1974). Measurement of pessimism, the Beck Hopelessness Scale. Journal of Consulting and Clinical Psychology, 42, 861-865.

Bhar, S., Ghahramanlou-Halloway, M., Brown, G. \& Beck, A. (2008). Self esteem and suicide ideation in psychiatric outpatients. Suicide \& Life-Threatening Behavior, 38, 511- 516.

Bisquerra, R. (1989). El análisis multivariante. Barcelona: PPU.

Cabrera, R. \& Castillo, L. (2008). Validación de un modelo de factores protectores frente a la ideación suicida en adolescentes, estudiantes de bachillerato, de la ciudad de San Juan de Pasto. Trabajo de grado para optar al título profesional en Psicología, Manuscrito no publicado, Universidad de Nariño, Pasto, Colombia.

Chioqueta, A. \& Stiles, T. (2007). The relationship between psychological buffers, hopelessness and 
suicidal ideation. Identification of protective factors. Crisis, 28, 67-73.

Clum, G., Canfield, D., Arsdel, M., Yang, B., Febbraro, G. \& Wright, J. (1997). An expanded etiological model for suicide behavior in adolescents: Evidence for its specificity relative to depression. Journal of Psychopathology and Behavioral Assessment, 19, 207-222.

De Leo, D., Burgis, S., Bertolote, J., Kerkhof, A. \& BilleBrahe, U. (2004). Definitions of suicidal behaviour. En D. De Leo, U. Bille-Brahe, A. Kerkhof \& A. Schmidtke (Dirs.), Suicidal Behaviour. Theories and Research Findings (pp. 17-40). Göttingen: Hogrefe \& Huber Publishers.

Field, T., Diego, M. \& Sanders, C. (2001). Adolescent suicidal ideation. Adolescence, 36, 241-248.

González O. (2007). Epidemiología de los suicidios. Colombia, 2007. En Instituto Nacional de Medicina Legal y Ciencias Forenses, Forensis 2007. Datos para la vida (pp. 179-204). Consultado el 15 de abril, 2009 en http://www.medicinalegal.gov.co/ drip/2007/\%20Forensis\%202007\%20suicidio. df-1.pdf

González-Forteza, C. (1992). Estresores psicosociales y respuestas de enfrentamiento en los adolescentes: Impacto sobre el estado emocional. Tesis para obtener el grado de Maestría en Psicología Social, Facultad de Psicología, UNAM, México.

González-Forteza, C., García, G., Medina-Mora, M. \& Sánchez, M. (1998a). Indicadores psicosociales predictores de ideación suicida en dos generaciones de estudiantes universitarios. Salud Mental, 21, 1-9.

González-Forteza, C., Mariño, M., Rojas, E., Mondragón, L. \& Medina-Mora, M. (1998b). Intento de suicidio en estudiantes de la Ciudad de Pachuca, Hgo. y su relación con el uso de sustancias y el malestar depresivo. Revista Mexicana de Psicología, $15,165-175$.

González-Forteza, C., Ramos, L., Caballero, M. \& Wagner, F. (2003). Correlatos psicosociales de depresión, ideación e intento suicida en adolescentes mexicanos. Psicothema, 15, 524-532.

González-Forteza, C., Villatoro, J., Alcántara, I., Medina-Mora, M., Fleiz, C., Bermúdez, P. \& Amador, N. (2002). Prevalencia de intento suicida en es- tudiantes adolescentes de la Ciudad de México: 1997-2000. Salud Mental, 25, 1-12.

González-Macip, S., Díaz, A., Ortiz, S., González-Forteza, C. \& González-Núñez, J. J. (2000). Características psicométricas de la Escala de Ideación Suicida de Beck (ISB) en estudiantes universitarios de la ciudad de México. Salud Mental, 23, 21-30.

Groholt, B., Ekenberg, O., Wichstrom, L. \& Haldorser, T. (2005). Suicidal and non-suicidal adolescents: Different factors contribute to self-esteem. Suicide and Life-Threatening Behavior, 35, 525-535.

Gutiérrez, P., Osman, A., Barrios, F. Kopper, B., Baker, M. \& Haraburda, C. (2002). Development of the Reasons for Living Inventory for young adults. Journal of Clinical Psychology, 58, 339-357.

Haseitel, B., Sáenz, C., Pérez, L. \& Haseitel, M. (2004). "Suicidio en Corrientes Capital": predominio en adolescentes y adultos jóvenes. Revista de Postgrado de la VI Cátedra de Medicina, 138, 7-10. Consultado el 15 de noviembre, 2007 en http:// med.unne. edu.ar/revista/ revista138/scc.pdf

Instituto de Investigación y Desarrollo en Prevención de Violencia y Promoción de la Convivencia Social - CISALVA. (2008). Guía metodológica para la replicación de observatorios municipales de violencia. Cali: CATORSE CS. Consultado el 17 de abril, 2009 en http://www.cepis.org.pe/bvsdevio/ fulltext/Guia_Metodologica.pdf

Konick, L. \& Gutiérrez, P. (2005). Testing a model of suicide ideation in college students. Suicide and Life-Threatening Behavior, 35, 181-192.

Lieberman, Z., Solomon, Z. \& Ginzburg, K. (2005). Suicidal ideation among young adults: Effects of perceived social support, self esteem and adjustment. Journal of Loss and Trauma, 10, 163-181.

Medina-Mora, M., López, E., Villatoro, J., Juárez, F., Carreño, S., Berenzon, S. \& Rojas, E. (1994). La relación entre la ideación suicida y el abuso de sustancias. Resultados de una encuesta en la población estudiantil. Anales del Instituto Mexicano de Psiquiatría, 6, 7-14.

Mingote, J., Jiménez, M., Osorio, R. \& Palomo, T. (2004). Suicidio: asistencia clínica. Guía práctica de psiquiatría médica. Madrid: Díaz de Santos.

Montero, I. \& León, O. (2007). Guía para nombrar los estudios de investigación en Psicología. Inter- 
national Journal of Clinical and Health Psychology, 7, 847-862.

Muehlenkamp, J., Gutiérrez, P., Osman, A. \& Barrios, F. (2005). Validation of the Positive and Negative Suicide Ideation (PANSI) Inventory in a diverse sample of young adults. Journal of Clinical Psycho$\log y, 61,431-445$.

Organización Mundial de la Salud. (2003). Informe Mundial sobre la Violencia y la Salud. Washington: Autor.

O Carroll, P., Berman, A., Maris, R., Moscicki, E., Tanney, B. \& Silverman, M. (1996). Beyond the Tower of Babel: A nomenclature for suicidology. Suicide and Life-Threatening Behavior, 26, 237-252.

Osman, A., Barrios, F., Gutiérrez, P., Wrangham, J., Kopper, B., Truelove, R. \& Linden, S. (2002). The Positive and Negative Suicide Ideation (PANSI) Inventory: Psychometric evaluation with adolescent psychiatric inpatient samples. Journal of Personality Assessment, 79, 512-530.

Osman, A., Gutiérrez, P., Jiandani, J., Barrios, F., Linden, S. \& Truelove, R. (2003). A preliminary validation of the Positive and Negative Suicide Ideation (PANSI) Inventory with normal adolescent samples. Journal of Clinical Psychology, 59, 493-512.

Osman, A., Gutiérrez, P., Kopper, B., Barrios, F. \& Chiros, C. (1998). The positive and negative suicide ideation inventory: Development and validation. Psychological reports, 82, 783-793.

Pollock, L. \& Williams, J. (2004). Problem-solving in suicide attempters. Psychological Medicine, 34, 163-167.

Radloff, L. S. (1977). The CES-D Scale: A self report depression scale for research in the general population. Journal of Applied Psychological Measurement, 1, 385- 401 .
Roberts, E. (1980). Reliability of the CES-D Scale in different ethnic contexts. Psychiatry Research, 2, 125-134.

Rodríguez, J., Fernández, A., Hernández, E. \& Ramírez, S. (2006). Conductas agresivas, consumo de drogas e intentos de suicidio en jóvenes universitarios. Terapia Psicológica, 24, 63-69.

Rosenberg, M. (1965). La autoimagen del adolescente y la sociedad. Buenos Aires: Paidós.

Schotte, D. \& Clum, G. (1987). Problem solving skills in suicidal psychiatric patients. Journal of Consulting and Clinical Psychology, 55, 49-54.

Siegel, S. \& Castellán, N. (2007). Estadística no paramétrica aplicada a las ciencias de la conducta (4ª ed.). México: Trillas.

Vázquez, A., Jiménez. R. \& Vázquez, R. (2004). Escala de autoestima de Rosenberg: fiabilidad y validez en población clínica española. Apuntes de Psicología, 22, 247-255.

Villalobos-Galvis, F. (2006). Validación de instrumentos de evaluación psicológica en estudiantes de la ciudad de San Juan de Pasto. Universidad de Nariño. Documento inédito.

Villalobos-Galvis, F. (2007). Aplicación del modelo procesual del estrés a la conducta suicida. Universidad y Salud, 8, 126-141.

Villalobos-Galvis, F. (2009). Situación de la conducta suicida en estudiantes de colegios y universidades de San Juan de Pasto. Salud Mental, 32 (2), 165-172.

Villalobos-Galvis, F. (2010). Estructura factorial y propiedades psicométricas de una escala de depresión en universitarios de Colombia. Revista Panamericana de Salud Pública, 27(2), 110-116.

Wild, L., Flisher, A. \& Lombard, C. (2004). Suicidal ideation and attempts in adolescents: Associations with depression and six domains of self-esteem. Journal of Adolescence, 27, 611-624. 